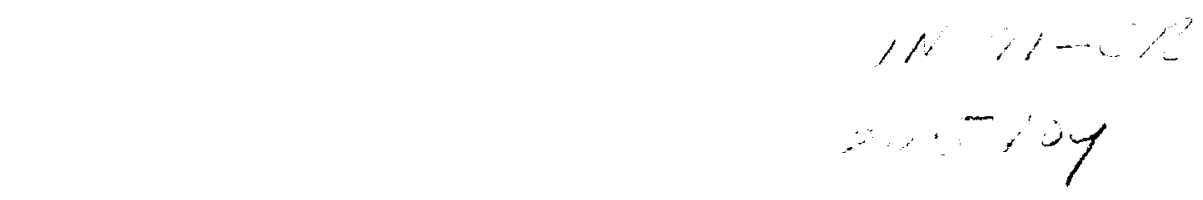

\title{
MICRO WEATHER STATIONS FOR MARS
}

\author{
David Crisp, Jet Propulsion Laboratory (JPL), \\ William J. Kaiser (JPL), Thomas R.VanZandt (JPL), \\ Michael E. Hoenk (JPL), James E. Tillman (University of Washington)
}

\begin{abstract}
:
A global network of weather stations will be needed to characterize the near-surface environment on Mars. Here, we review the scientific and measurement objectives of this network. We also show how these objectives can be met within the costconstrained Mars Surveyor Program by augmenting the Mars Pathfinder-derived landers with large numbers of very small ( $<5$ liter), low-mass ( $<5 \mathrm{~kg}$ ), low-power, low-cost Mini-Met Stations. Each station would include instruments for measuring atmospheric pressures, temperatures, wind velocities, humidity, and airborne dust abundance. They would also include a data handling, telemetry, power, atmospheric entry, and deployment systems in a rugged package capable of direct entry and a high-impact landing. In this paper, we describe these systems and summarize the data-taking strategies and data volumes needed to achieve the surface meteorology objectives for Mars.
\end{abstract}

\section{INTRODUCTION:}

The thermal structure and dynamics of the Martian atmosphere are strongly influenced by the exchange of heat, mass, and momentum between the surface and atmosphere, through the planetary boundary layer (PBL). A comprehensive description of the meteorology near the Martian surface is therefore essential for an improved understanding of the Martian climate and general circulation. A more complete characterization of the near-surface meteorology is also needed for future robotic and manned missions to this planet because the surface will be their working environment. The only existing in-situ Martian meteorological measurements were collected by the two Viking Landers. These stations measured large variations in the atmospheric pressure ( 7 to $10 \mathrm{mb})$, temperature $(170$ to $220 \mathrm{~K})$, wind velocity $(0$ to $25 \mathrm{~m} / \mathrm{sec})$, and airborne dust optical depth $(0.3$ to $>4$ ) on diumal and seasonal time scales (Zurek et al. 1992). Remote sensing observations suggest that an even wider range of conditions exist at some latitudes and seasons. The principle shortcoming of the Viking meteorological measurements is their limited spatial coverage (only 2 sites). In addition, even though Viking Lander 1 acquired data for about two Martian years, its measurements provide too litue temporal coverage to adequately describe the mean climatology or the interannual variability of the Martian atmosphere.

The principal science objectives for future Mars meteorological measurements were summarized in the 1990 NASA Ames Network Meteorology Workshop Report (R. Haberle, 1990). These objectives can be summarized as follows: (i) to describe the temporal and spatial structure of the general circulation, including the zonal mean circulation (Hadley cell, $\mathrm{CO}_{2}$ mass flow). planetary-scale eddies (thermal tides, Kelvin waves), and small scale features (slope winds, gravity waves), (ii) to understand the relationship between the circulation and its forcing (radiative, and latent heating., and forcing associated with albedo, thermal inertia, roughness, topography), and external factors (planetary radius, rotation, gravity), and (iii) to relate that understanding to the climate system, in general, including the transport and deposition of dust and volatiles, aeolian modification of the surface, and sources and sinks of water and $\mathrm{CO}_{2}$ (polar caps, regolith)

A comprehensive measurement campaign by a global network of surface stations, accompanied by remote-sensing instruments on orbiters would be needed to address all of these objectives. The in-situ surface measurements complement observations obtained by orbiters by providing ground-truth. as well as a more comprehensive and reliable description of the meteorology within the atmospheric boundary layer. A detailed understanding of the near-surface meteorology is also needed for the analysis surface geophysical and geochemical measurements. For example, wind measurements will be needed in conjunction with seismological measurements because surface winds will provide the principal source of seismic noise. In addition to these scientific objectives, there are several purely-programmatic reasons for studying the meteorology near the Martian surface. The planetary boundary layer will be the working environment for future robotic and manned missions to Mars. The large $(-100 \mathrm{~K})$ diumal and seasonal temperature variations can affect lander system performance and calibration. High winds and low-humidity conditions at some sites will encourage static buildup. Wind-blown sand and dust that can cause abrasion to sensors and other hardware, and dust accumulation. which can reduce the efficiency of solar arrays, and alter the electrical and thermal properties of instruments and spacecraft systems. 
Our next opportunity to study the near-surface environment on Mars will be provided by the Mars Surveyor Program. As currently conceived, this program will deploy one or more Mars Pathfinder-derived landers at each launch opportunity, beginning in 1998. We anticipate that each of these landers will include meteorological instruments as well as instruments for studying the geology, geochemistry, and. geophysics of the Martian surface. It will be impossible to deploy a global network of these relatively large, complex, expensive landers within the scope of this cost-constrained program. Fortunately, the meteorological objectives described above could be satisfied by replacing one of the Pathrinder-derived landers or augmenting these landers with large numbers (dozens) of small ( 2 to $5 \mathrm{~kg},-5$ liter), low-cost ( 1 to 5 M\$) autonomous meteorology stations. Each of these Mini-Met Stations should include instruments for measuring the atmospheric pressure. temperature, wind velocity, humidity, and airbome dust abundance. In addition, each lander must include data handling, telemetry, power, atmospheric entry, landing, and instrument deployment systems Meteorological instruments like those used on Viking and Mars Pathfinder are not well suited for this application because they are too large, too massive, too fragile, or require too much power for deployment on these very small platforms. Fortunately, there have been a number of recent advances in sensors, electronics, and materials that provide dramatic improvements in sensor capability, and durability, while facilitating the reductions in size, mass, and cost needed to satisfy the stringent constraints on this program.

Our team has been studying the feasibility and utility of miniaturized meteorological sensors for in-situ measurements at the Martian surface. Our goal was to develop rugged, compact, light-weight, and low-power weather stations that would place a minimum burden on lander resources. We also focused on sensor technologies that could be calibrated remotely, or those that featured long-term calibration stability so that they would continue to return useful data for several Martian years in an environment characterized by diumal and seasonal temperature variations as large as $100 \mathrm{~K}$, high winds, and varying dust amounts. Many of the technologies needed to satisfy these requirements exist, and can obtained commercially. For example. the pressure sensors and airborne dust optical depth instruments developed for the Russian Mars 94/96 Missions are ideal for these small stations. Thermocouples, like those used for atmospheric temperature measurements by the Viking Lander, can be used by the proposed small stations as well. Existing commercially-available sensors for measuring other meteorological parameters, including wind velocity and humidity, do not provide the sensitivity, accuracy, speed, or long-term calibration stability needed for Mars surface applications. Our team has therefore combined novel measuring techniques with silicon micro-machined structures to develop miniaturized anemometers and hygrometers.

In addition to these sensor development activities, we have initiated a study of the data handling, telemetry, power, and deployment systems needed for these stations. We have not yet completed the integration of these systems, but we have developed a conservative design based on the sensors described above. their deployment hardware ( 2 short booms), a meteorological data logger (sensor analog electronics. AVD converter, CPU, RAM, EEPROM, and associated digital electronics), and a $403 \mathrm{MHz}$ transmitter compatible with the Mars Balloon Relay for transmission of the data ( $50 \mathrm{Kbits} / \mathrm{Sol}$ ) to Mars orbit. This entire system fits in a 0.6 liter package and has a mass of only $0.9 \mathrm{~kg}$. Substantial reductions in the size and mass of the electronics could be achieved by wider use of Application Specific Integrated Circuits (ASICs) and multi-chip modules. This system requires an estimated $300 \mathrm{~mW}$ for continuous operation (not counting telemetry), but we anticipate that it will be operated with a $1 / 10$ duty cycle ( 6 minutes/hour). The power system consists of rechargeable batteries and either a modified Radioisotope Heating Unit (RHU - essential for continuous high-latitude operation) and/or a small ( $30 \mathrm{by} 30 \mathrm{~cm}$ ) solar array. Here, we will describe the current status this system slong with its deployment and performance requirements. We will also summarize the data-taking strategies and dais volumes required to achieve the science objectives for surface meteorology on the Surveyor mission.

\section{SURFACE METEOROLOGICAL MEASUREMENTS NE.EDED:}

The meteorological quantities that can be measured by small. low-pxwer. surface stations include atmospheric pressure, temperature, winds, humidity, and airbome dust and ice amounts. These quatntities are listed in Table 1, along with estimates of the dynamic range, resolution and accuracy needed, and data volume required. Several sampling times are included for each measurement type, along with a brief description of the science issue that is addressed by that sampling rate. The science return is proportional to the sampling rate, and the total volume of science data that is returned by the Mini-Met network. Constraints on the available development time and cost, as well as limitations in the on-board power (for instruments and the data processing) and telemetry may preclude meeting all of these objectives. We have therefore prioritized these measurements based on the expected science renurn (within these constraints), and listed them in order of decreasing priority. 
INSTRUMENT TYPES AND DEPLOYMENT STRATEGIES:

Pressure: Surface pressure measurements have been given the highest priority by Mars science working groups because they provide a means to monitor the climate and total mass of the entire Martian atmosphere from a single surface site. Pressure variations also indicate the passage of local weather systems. and provide constraints on the atmospheric dust loading. The pressure sensor can be located within the lander, and connected to the environment through a port. No special deployment mechanisms or orientation is needed for surface pressure measurements. The micro-machined Vaisalla Barocaps that are being used on the Russian Mars 94 Small Stations and the Mars Pathfinder have adequate dymamic range $(0$ to $50 \mathrm{mb}$ ), accuracy $(0.02 \mathrm{mb})$, resolution $(0.005 \mathrm{mb})$ and calibration stability for this application (Table 1). Their mass is only 15 gms (with readout electronics), their volume is about $1 \mathrm{cc}$, and they require only $10 \mathrm{~mW}$ of power for continuous operation.

Column Optical Depth/Sky Brightness: The absorption of sunlight by airbome dust provides the principle source of heating in the Martian atmosphere. Measurements of the atmospheric dust loading can also be combined with temperature, pressure, and wind measurements for studies of dust storm genesis. Detailed studies of the effects of airborne dust on the Martian atmosphere will require measurements of the verical distribution of the dust, as well as its total column abundance. Measurements of the dust vertical distribution can best be obtained by limb-scanning instruments on orbiters. The columnintegrated dust optical depth can be determined from surface measurements of the attenuation of sunlight or the light from other astronomical objects (Pollack et al. 1979, Pollack 1982). A well-calibrated. multi-spectral camera would provide the best approach for monitoring the column optical depth and sky brightness, but this system must be capable of scanning from the horizon to the zenith. If a system like this cannot be produced within the mass and power constraints of these small stations, a much simpler optical sensor could be used for this application. For example, L. Esposito and his colleagues at LASP (U. Colorado) have developed an optical depth sensor based on a diode array for use on the Russian Mars $94 / 96$ penetrators. This system has a mass of less than $50 \mathrm{gms}$, and requires less than $50 \mathrm{~mW}$ for continuous operation, and could still satisfy the specifications listed in Table 1.

Temperature: Temperature measurements from a surface station could provide improved constraints on the near-surface atmospheric temperatures, which are very difficult to infer by remote-sensing methods. These measurements could yield insight into the diurnal temperature cycles (surface and atmospheric thermal inertia), the boundary layer stability, and the heat and mass transport at that site. Accurate atmospheric temperature measurements are somewhat challenging in the thin Martian atmosphere, were solar and thermal radiative heating of the sensor element, and conduction losses to the sensor support structure can introduce significant sources of error. Fortunately, the 75- $\mu \mathrm{m}$ thermocouples, like those used on the Viking Landers, provide adequate accuracies for this application. These sensors and their associated electronics can now be produced with dimensions and power requirements similar to those of the Barocaps described above. Atmospheric temperatures should be measured from a boom that extends as far away from the station as possible to minimize the effects of its thermal plume. Although long booms are desirable. much shore booms $(-30 \mathrm{~cm})$ would be adequate if the lander is very small. One simple way to minimize the thermal impact of the station is to deploy temperature sensors from two booms. that extend away from different sides of the station to form a " $V$ ". In this configuration, the thermal plume from the station will miss both temperature booms when there is no wind, or when the wind blows perpendicular to the plain defined by the two booms, and only one boom is affected if winds blow parallel to plane of the two booms. Surface and near-sub-surface $(1-10 \mathrm{~cm})$ temperature measurements would also be extremely valuable for studies of surface-atmosphere interactions, and surface thermal inertia.

Wind: Wind measurements obtained from a surface lander would facilitate studies of the exchange of heat, mass. and momentum between the surface and the atmosphere. Wind direction is somewhat more difficult to measure than wind speed. but accurate estimates of both parameters are needed to estimate fluxes. Much less accuracy is needed to estimate the surface wind stress, however, and improved estimates of this quantity are needed for atmospheric modeling studies. In addition, if wind measurements could be correlated with measurements of the local particle number density. they could be used for studies of saltation rates and aeolian transport. We are developing two different wind-measuring concepts. The first is a single-chip, micro-machined thermal anemometer. This sensor consists of an interdigitated heater and platinum resistance thermorneter suspended on a thin $(-5 \mu \mathrm{m})$, low-thermal-inertia silicon nitride membrane micro-machined into the center of a small ( 1 by $1 \mathrm{~cm}$ ) silicon structure. The heated membrane is surrounded on 4 sides by platinum resistance thermometers, each of which is supported on its own thin nitride membrane. As wind blows over the sensor, the wind speed is determined from the amount of power needed to maintain the heated membrane at a fixed over-temperature (as in a conventional hot-film anemometer). The wind direction is determined by comparing the temperatures measured by the 4 surrounding temperature sensors. This sensor is best suited for measuring very low wind speeds $(1 \mathrm{~cm} / \mathrm{sec}$ to $\sim 10 \mathrm{~m} / \mathrm{sec})$. A second sensor is under development 
for measuring higher wind speeds. This is a pitot-static anemometer, that measures wind velocities by measuring the deflections of a thin membrane. In this system. membrane deflections produce variations in capacitance between an electrode on the membrane and a fixed electrode. This system is based on a version of the a high-frequency capacitive circuit that was developed for the JPL Micro Seismometer (Banerdt et al. this meeting). This system can detect membrane deflections as small as a few angstroms.

Like temperatures, atmospheric winds should be measured from booms that extend as far away from the lander as possible to minimize the dynamical effects of the lander. If possible, a second wind measurement should be made at or near the ground for studies of dust saltation.

Humidity: The Viking Landers did not include humidity sensors, but Viking Orbiter observations (Farmer et al. 1979) indicated large variations in the atmospheric water abundance. Accurate measurements of humidity variations over diurnal and seasonal cycles will yield valuable new constraints on moisture sources and sinks, as well a the exchange of moisture between surface and atmospheric reservoirs. The Russian Mars 94 Small Stations will use Vaisalla Humicaps for measuring the humidity near the Martian surface. These determine the humidity by measuring capacitance variations associated with the adsorption of water by a polymer. They are sensitive at temperatures as low as $-70 \mathrm{C}$, but their time constants exceed 1000 seconds at those low temperatures (A.-M. Harri. 1993). The long-term calibration stability of these devices is also unknown in the dusty Martian atmosphere. To improve the sensitivity to small water concentrations at low temperatures, reduce the response time, and reduce the chances of long-term calibration shifts, we have developed a series of miniature hygrometers that determine humidity by measuring the dew point temperature. These sensors consist of small ( 5 cubic $\mathrm{mm}$ ) proprietary transducers that are cooled from the ambient temperature through the dew point by a miniature thermo-electric cooler (TEC). Comparisons between these sensors and NST-traceable standards confirm that they are sensitive to sub-monolayer water abundances. This should allow fast, accurate measurements of water amounts in the neighborhood of one part in $10^{7}$ in the Marian atmosphere. Because the transducers are very small, little power is needed to cycle them rapidly $(-1 \mathrm{sec} / \mathrm{K})$ over a wide range of dewpoint temperature depressions. In addition, our tests have shown that they are very resistant to calibration shifts caused by dust and other sources of contamination. These humidity sensors can be mounted on the body of the lander, or on a mast, but they should be located as far as possible from major heat sources and potential moisture reservoirs. In addition, because these sensors are cooled, they should not be located near the temperature sensors.

Column Water Vapor Sensor: Measurements of the column-integrated water abundance would provide a means of monitoring the large-scale transport of water vapor by the atmosphere. The column water abundance could be monitored during the day by measuring the intensity of sunlight at wavelengths in and out of near-infrared water bands at 0.94 or $1.33 \mu \mathrm{m}$. This sensor cold consist of three InGaAs diodes, or a small InGaAs diode array with narrow-band filters to isolate the water vapor band. This system could have a volume as small as 5 cubic centimeters, and a mass of less than 10 grams. These sensors could be located on the top of the lander, but they should have an unobstructed view of the sky.

Local Airborne Particle Detector: A paricle size spectrometer would provide the most comprehensive information about the dust abundance and size distribution near the lander, but even a simple backscatter nephelometer, consisting of a regulated light source and a detector could yield valuable information about dust cohesion and saltation and frost formation. This sensor could be located on the body of the lander body. The nephelometers flown on the Soviet/French/US Vega Balloons had masses near $100 \mathrm{gms}$, volumes of about $20 \mathrm{cc}$. and required about $100 \mathrm{~mW}$ of power for continuous operation. Recent advances in electronics could yield order-of-magnitude reductions in the size and mass of these systems. Their power consumption could by reduced by limiting their duty cycle.

\section{DATA SAMPLING STRATEGIES:}

The nature of atmospheric variability near the surface places a number of constraints on the sampling rates that are needed for each sensor type. For example, some meteorological quantities, like temperature and wind velocity can change dramatically on time scales as short as seconds. These quantities must be sampled rapidly enough to resolve these variations $(-1 \mathrm{~Hz})$, or the measurements will be badly aliased, and very difficult to interpret. The need for rapid sampling does not necessarily require large data rates, because long-duration averages of these variables, along with simple statistics (standard deviations, minimum and maximum values), can be extremely valuable. We therefore propose that temperature, wind velocity, and local dust abundance be sampled with a frequency of $1 \mathrm{~Hz}$. Some of this high-frequency data should be retumed to Earth, but limitations in data rate may preclude sending all of it back. The amount of data that must be retumed can be 
reduced substantially if these measurements can be linearized by the on-board processor and averaged for periods of several minutes to one hour. These averaged quantities could be augmented by simple statistics, including their standard deviation. minimum, and maximum values. It would also be useful to compute and transmit correlations between the wind velocity and the temperature (heat flux), or the local particle concentration (saltation events). It would be best to measure temperature, wind velocity, and local particle abundance continuously. but limitations in on-board power may require that the duty cycle be reduced to a value as low as $10 \%$ (ie. 6 minutes per hour). Other quantities such as the pressure, humidity, airbome dust and ice loading, and the solar and thermal radiances could be sampled much less frequently. For example, these instruments could be activated for as little a few seconds each half-hour.

\section{DATA VOLUME:}

The total data budget of these stations includes the meteorological measurements described above, along with the timing and engineering data needed to interpret these measurements. We anticipate that each lander will store its measurements for one or more days, and then transmit them to an orbiter when it receives a coded beacon.

Science Dala: The volume required for routine measurements by each of the 9 meteorological sensor types is itemized in Table 1 ( $-20.6 \mathrm{Kbit} / \mathrm{day})$. For Pressure, temperature, wind velocity, and particle backscatter, this data volume estimate includes the minimum, maximum, mean, and standard deviation for each half-hour sampling interval. For the column optical depth measurement, it includes the first 32 modes of the intensity distribution. taken at half-hour intervals during the day. It should be emphasized that any enhancement in these data volume estimates could yield substantial improvements in the science rearn. In addition to these routine samples, short segments of the high-resolution temperature and wind (and perhaps nephelometer) time series should be sent back occasionally to confirm their calibration. Calibration data from other sensors will also be needed occasionally. Here, we assume that the duration and frequency of these calibration data sets is chosen to be roughly comparable to that produced by the routine sampling ( $20.6 \mathrm{Kbit/day})$

Timing dara: If measurements are reported every half-hour, and labeled with a simple 12-bit time tag, the timing data will require an additional $0.6 \mathrm{Kbi} / \mathrm{day}$.

Engineering Data: The amount of engineering data will depend on the details of the meteorological instrumentation that is chosen, as well as the engineering requirements of the other systems included in the station (e.g. power, telemetry). For example, each meteorological sensor might require one or more internal temperature or electrical (e.g. voltage, current) measurements ( $4.8 \mathrm{Kbi} / \mathrm{day}$ ) which must be returned with each routine sample. The power system and transmitter will also return some status information ( $1.2 \mathrm{Kbiv/day})$. These data volume requirements are summarized in Table 2 . Once again, we must emphasize that these are the minimum requirements for each lander, and that the science return of mission could be substantially increased by increasing these estimates.

\section{PROPOSED SYSTEM CONFIGURATIONS:}

We have developed a minimum system, which includes the meteorologicai sensors, a data handling system, a transmitter, a power system. and instrument deployment hardware, consisting of two small $(30 \mathrm{~cm}$ to $1 \mathrm{~m})$ booms. The components of these systems are listed in Table 3, along with very conservative estimates of their required volume, mass, and power. The data system adopted for the Mini-Met Station has capabilities similar to those of conventional. commercially-available meteorological data logger/instrument controllers, like those marketed by Campbell Scientific Inc (c.f. Fredrickson. 1993). The Mini-Met data system includes (i) the analog electronics for all instruments except the barometer, (ii) a 14-bit $A / D$ converter, (iii) a micro-controller-based CPU, RAM, EEPROM, and VO subsystems. The telemetry system provides communications to a Mars orbiter. It employs a $403 \mathrm{MHz}$ transmitter compatible with the Mars Balloon Relay protocol, developed for the Mars $94 / 96$ mission. We anticipate that all future Mars orbiters will include receivers that are compatible with this protocol. The antenna is a $30 \mathrm{~cm}$ wire which will be incorporated into one of the two small masts. The power system consists of rechargeable batteries with one of two different battery recharging systems. For landers deployed at latitudes less than 60 degrees, small $(30$ by $30 \mathrm{~cm}$ ) solar panels will provide adequate power. At higher latitudes, it will be necessary to replace these solar cells with a mini-Radioisotope Thermoelectric Generator (RTG), or "Power Stick" consisting of a standard. space-qualified Radioisotope Heating Unit (RHU) and a thermocouple. In addition to these systems, each Mini-Met Station requires an entry system capable of direct. high-velocity entry into the Martian atmosphere, and a highimpact landing on the surface. This entry system has not yet been designed, but we anticipate that the total entry mass of each station will be much less than $5 \mathrm{~kg}$. and that their volumes will be less than 5 liters. 


\section{CONCLUSIONS:}

We have summarized the scientific and measurement objectives for Mars surface meteorology, and described an approach that employs a global network of Mini-Met stations to satisfy these requirements. Each station includes instruments for measuring pressure, temperature, wind velocity, humidity, and the airborne dust abundance. They also include a datahandling system based on a meteorological data logger/instrument controller, a telemetry system capable of transmitting data to a spacecraft in orbit around Mars, and a power system, consisting of rechargeable batteries and either a modified Radioisotope Heating Unit (RHU) (for year-round high-latitude operation) or a small ( $30 \mathrm{~cm}$ by $30 \mathrm{~cm}$ ) solar array. Because the sub-systems included in these stations are inherently rugged, they could be incorporated into an entry system capable of large decelerations and a high-impact landing ( $>1000$ 's $\mathrm{g}$ 's). With these characteristics, large numbers of these stations could be transported to Mars with a dedicated launch, or even as augmentations to other Surveyor payloads. They could be targeted to a wide range of latitudes by a spin-release mechanism on the carrier spacecraft, similar to that used on the Pioneer Venus Multi-Probe Mission. If such a system can be deployed, we could recover the global meteorological and climatological objectives of the MESUR mission at a small fraction of the cost.

\section{REFERENCES CITED:}

Frederickson. Eight Symposium on Meteorological Observations and Instumensation. American Meteorological Society. 219 224, 1992.

Pollack, J. B. et al., J. Geophys. Res. 84, 2929-2945, 1979.

Pollack, J. B. Adv. Space Res. 2 45-56. 1982.

Zurek, R. W. et al. Mars, University of Arizona Press, 835-933, 1992. 


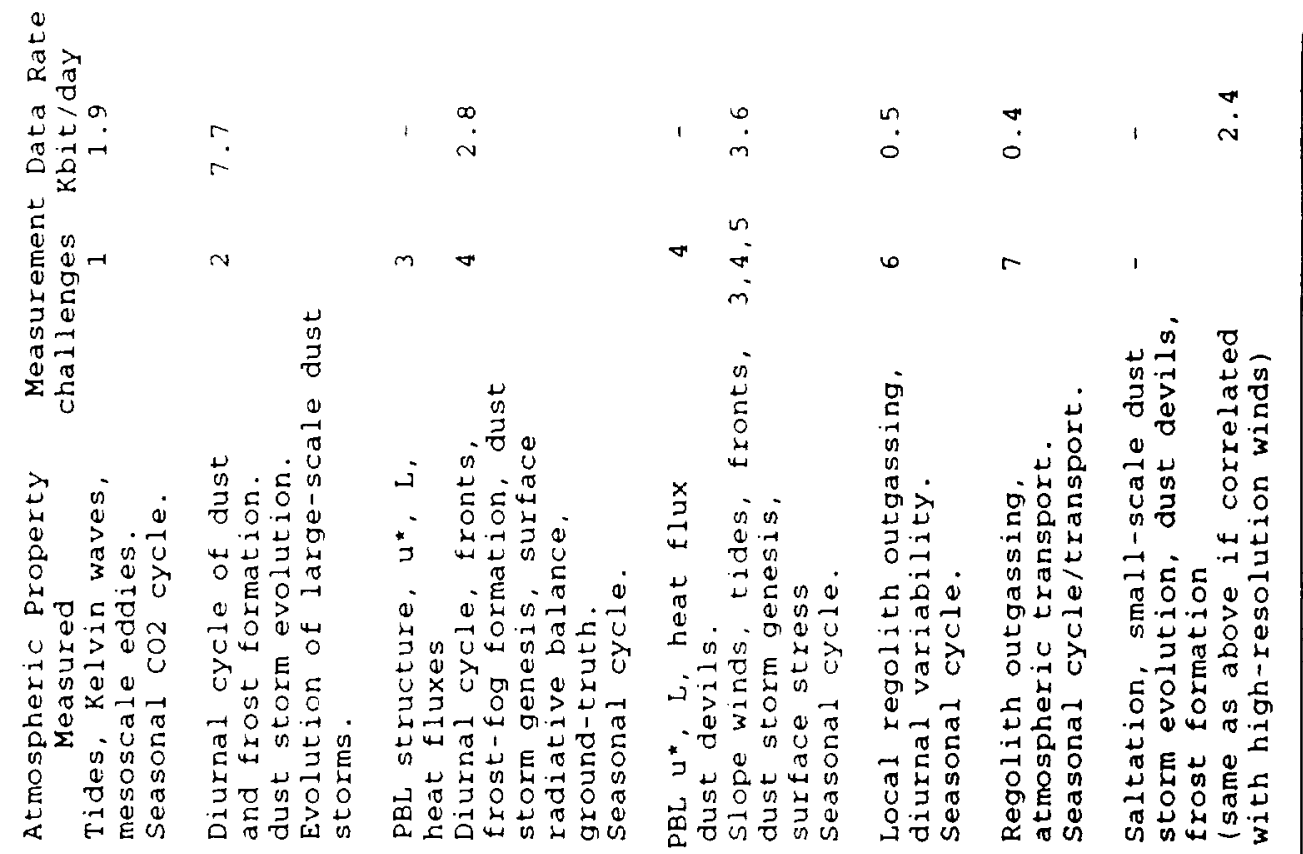

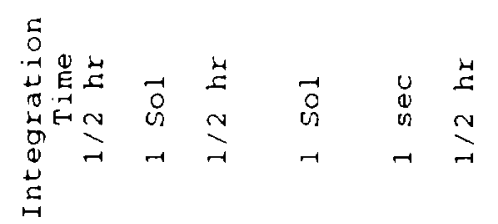
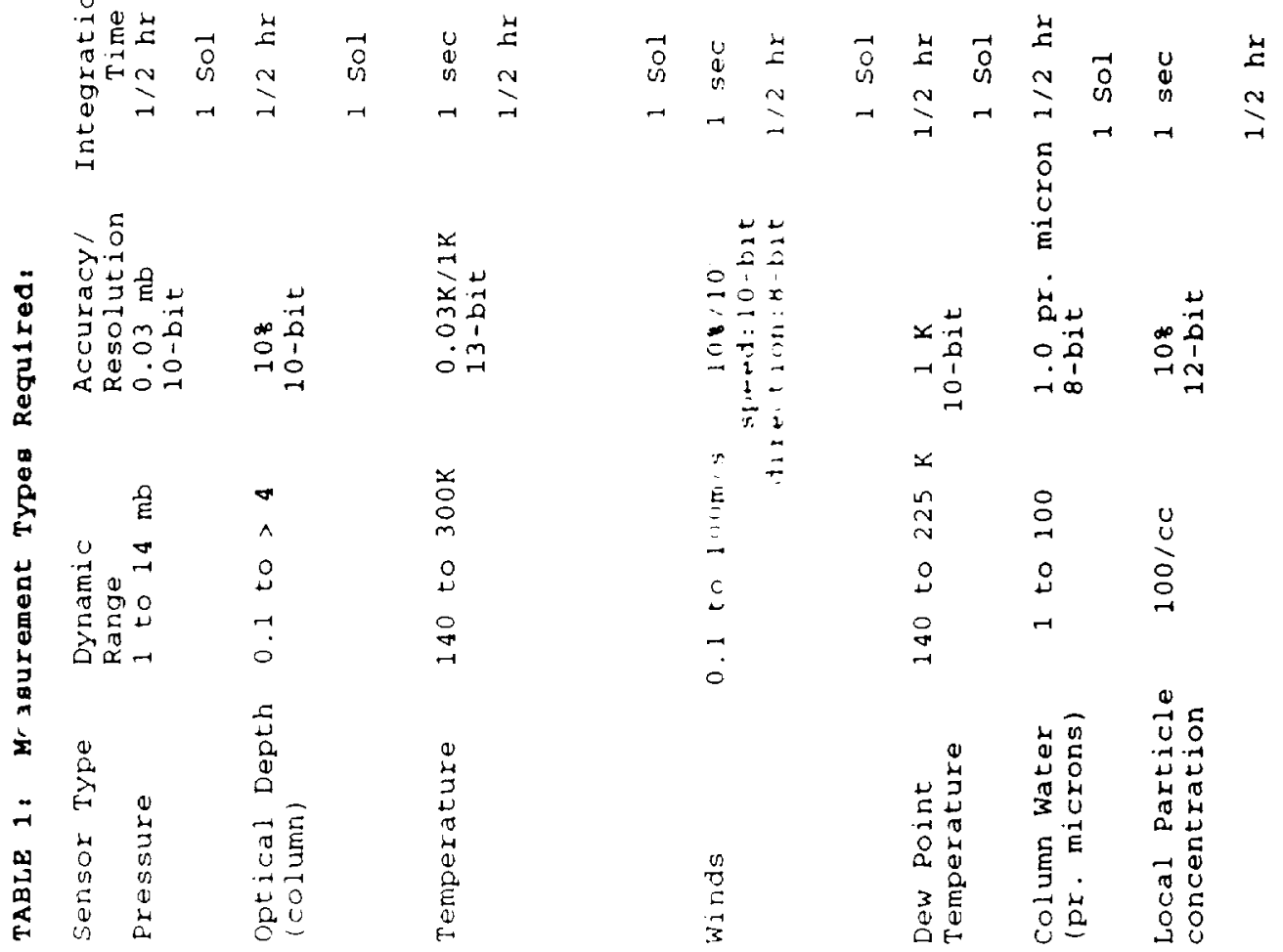


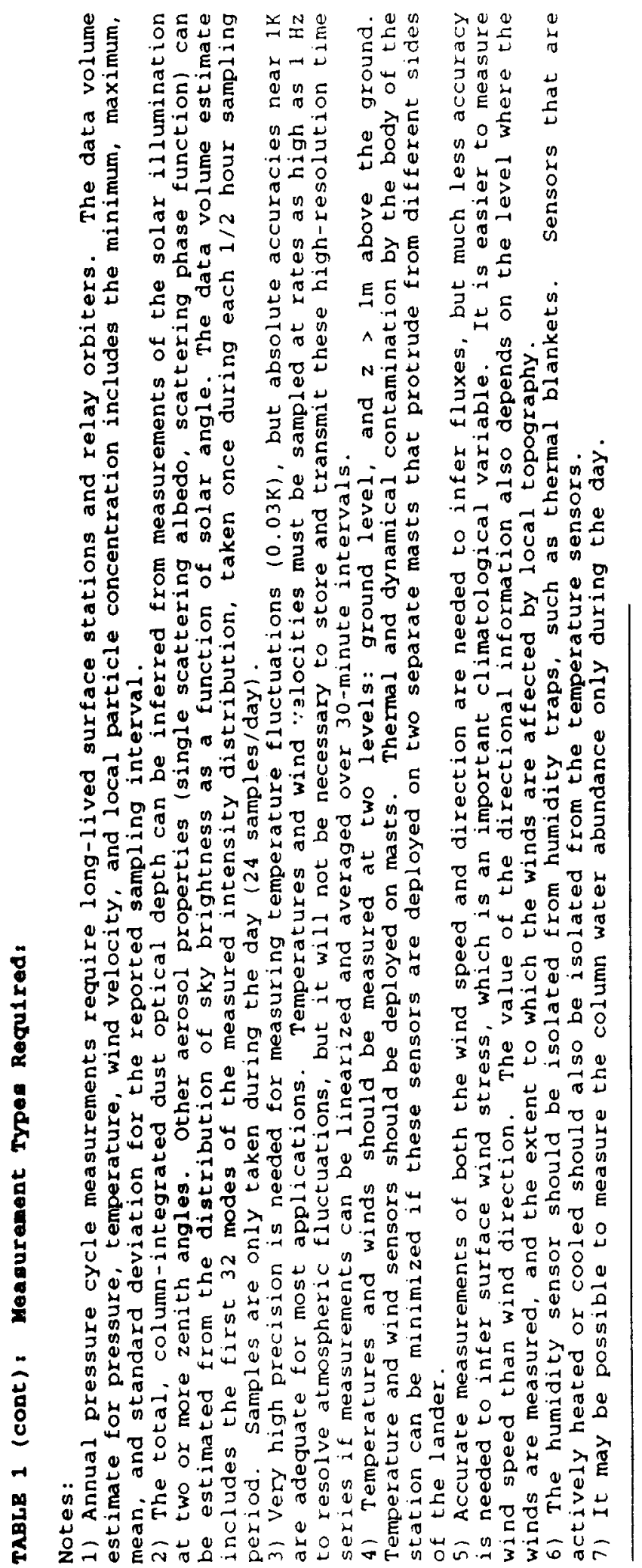


Table 2: Estimated Data Volumes for Each Station:

Routine Science Measurements

Calibration Time Series

Time Stamp

Sensor Engineering Data

System Engineering Data

Total Data Volume
20. Kbil/day

20. Kbit/day

$-0.6 \mathrm{Kbi} / \mathrm{day}$

$-4.8 \mathrm{Kbi} / \mathrm{day}$

$\sim 1.2 \mathrm{Kbit} / \mathrm{day}$

$47 \mathrm{Kbit} / \mathrm{day}$

Table 3: Minimum System:

\begin{tabular}{|c|c|c|c|}
\hline System Component & ne(cc) & $\operatorname{Mass}(\mathrm{gm})$ & Power $(\mathrm{mW})$ \\
\hline $\begin{array}{l}\text { Aneroid barometer } \\
\text { (with analog electronics) }\end{array}$ & 20 & 50 & 10 \\
\hline Column Optical Depth Sensor & 100 & 100 & 30 \\
\hline Thermocouple Sensors & 40 & 25 & 6 \\
\hline Anemometer & 40 & 25 & 30 \\
\hline Dew-Point Hygrometer & 20 & 10 & 100 \\
\hline Nephelometer & 70 & 100 & 50 \\
\hline Column Water Sensor & 50 & 100 & 25 \\
\hline $\begin{array}{l}\text { Two Temperature/Wind Sensor Booms } \\
\text { Booms (with deployment hardware) }\end{array}$ & 30 & 20 & - \\
\hline $\begin{array}{l}\text { Meteorological Data Logger } \\
\text { (CPU, Clock, RAM, EEPROM, 14-Bit AVD }\end{array}$ & 200 & 400 & 50 \\
\hline $\begin{array}{l}\text { Transmitter and Antenna } \\
\text { (Mars Orbit Only) }\end{array}$ & $<200$ & $<200$ & $<200$ \\
\hline $\begin{array}{l}\text { Power System (mini-RTG or Solar } 500 \\
\text { Cells and Batteries) }\end{array}$ & & 580 & -40 \\
\hline
\end{tabular}


